\title{
La violencia en las relaciones de pareja de jóvenes: prevalencia, victimización, perpetración y bidireccionalidad
}

\author{
Violence in the relationships of young couples: Prevalence, victimization, perpetration \\ and bidirectionality
}

\section{A violência nas relações de casais jovens: prevalência, vitimização, perpetração e bidirecionalidade}

\author{
María José Garrido Antón ${ }^{\mathrm{a}}$ |Ana Arribas Rey ${ }^{\mathrm{a}}$ | Jesús María de Miguel ${ }^{\mathrm{b}}$ | Ángel García-Collantes ${ }^{\mathrm{c}^{*}}$ \\ ${ }^{a}$ Guardía Civil, España \\ ${ }^{b}$ https://orcid.org/0000-0003-163 I-7670 Universidad Autónoma de Madrid, España \\ ' Universidad a Distancia de Madrid (UDIMA), España
}

- Fecha de recepción: 2020-03-12

- Fecha concepto de evaluación: 2020-04-13

- Fecha de aprobación: 2020-04-30 https://doi.org/I0.22335/rlct.vI2i2. II 68
Para citar este artículo / To reference this article / Para citar este artigo: Garrido Antón, M. J., Arribas Rey, A., de Miguel, J. M., \& García-Collantes, A. (2020). La violencia en las relaciones de pareja de jóvenes: prevalencia, victimización, perpetración y bidireccionalidad.

Revista Logos Ciencia \& Tecnología, I2(2), 8-19. https://doi.org/I0.22335/rlct.v12i2. I I 68

\section{RESUMEN}

El objetivo del trabajo fue estudiar las conductas violentas en las relaciones de pareja de jóvenes universitarios, así como la perpetración y la victimización de dichos comportamientos en función del sexo del sujeto que ejerce la violencia. Para esto se realizó un estudio transversal descriptivo sobre una muestra de estudiantes formada por 137 sujetos ( 101 mujeres y 36 hombres) con edades comprendidas entre los 18 y los 25 años. Los resultados indican que la violencia verbal era el subtipo de agresión más frecuente entre las parejas adolescentes con independencia del sexo. En relación con el tipo de violencia física - tanto ejercida como sufrida - no se hallaron diferencias significativas en función del género. Sin embargo, en el caso de las agresiones sexuales y los comportamientos de control se hallaron diferencias significativas entre mujeres y hombres. En general, el tipo de violencia que se detectó con más frecuencia en este tipo de relación fue de tipo bidireccional, usándose la violencia como método para resolver conflictos en la relación o como consecuencia de comportamientos de control y celos que se mostraron independientemente del sexo de los sujetos. Este tipo de investigaciones son importantes en el propósito de dirigir las políticas de prevención de la violencia y conseguir que se traduzcan en líneas de acción para la reducción o eliminación de esta tipología delictiva. Esto se traduciría en grandes beneficios para las víctimas directas e indirectas, en particular, así como para la sociedad en general.

Palabras clave: violencia bidireccional, violencia física, violencia verbal, violencia sexual, violencia en jóvenes

\footnotetext{
*Autor para correspondencia. Correo electrónico: angel.garcia.c@udima.es
} 


\begin{abstract}
The objective of the work was to study the violent behaviours in the relationships of young university students, as well as the perpetration and victimization of said behaviours based on the gender of the person who exercises the violence. For this, a descriptive cross-sectional study was carried out on a sample of students made up of 137 subjects ( $10 \mathrm{I}$ women and 36 men) with ages ranging between 18 and 25. The results indicate that verbal violence was the most frequent subtype of aggression among adolescent couples, regardless of gender. Regarding the type of physical violence -exercised as well as suffered - no significant differences were found according to gender. However, in the case of sexual assaults and controlling behaviours, significant differences were found between men and women. In general, the type of violence that was detected most frequently in this type of relationship was bidirectional, where violence is used as a way of resolving conflicts in a relationship or as a consequence of control and jealousy behaviours that were shown, regardless of the gender of the subjects. This type of research is important in order to direct violence prevention policies and ensure that they are translated into lines of action for the reduction or elimination of this criminal typology. This would convert into great benefits for direct and indirect victims, in particular, as well as for society in general.
\end{abstract}

Keywords: bidirectional violence, physical violence, verbal violence, sexual violence, violence in youth

\title{
RESUMO
}

O objetivo do trabalho foi estudar os comportamentos violentos nas relações de casais de jovens universitários, bem como a perpetração e vitimização desses comportamentos com base no sexo do sujeito que exerce a violência. Para isso, foi realizado um estudo descritivo transversal em uma amostra de estudantes composta por 137 pessoas ( $10 \mathrm{I}$ mulheres e 36 homens) com idades entre 18 e 25 anos. Os resultados indicam que a violência verbal foi o subtipo de agressão mais frequente entre casais adolescentes, independentemente do sexo. Quanto ao tipo de violência física - exercitada e sofrida - não foram encontradas diferenças significativas de acordo com o sexo. No entanto, no caso de agressões sexuais e comportamentos controladores, foram encontradas diferenças significativas entre mulheres e homens. Em geral, o tipo de violência detectada com mais frequência nesse tipo de relacionamento era bidirecional, usando a violência como um método para resolver conflitos na relação ou como consequência dos comportamentos controladores e ciúmes que eram mostrados independentemente do sexo do indivíduo. Esse tipo de pesquisa é importante para direcionar as políticas de prevenção à violência e garantir que elas sejam traduzidas em linhas de ação para a redução ou eliminação dessa tipologia criminal. Isso se traduziria em grandes benefícios para as vítimas diretas e indiretas, em particular, assim como para a sociedade em geral

Palavras-chave: violência bidirecional, violência física, violência verbal, violência sexual, violência em jovens

La violencia, en general, es un importante problema social presente, en gran medida, en cualquier sociedad, sin importar la clase social, la religión, el sexo, la edad, la raza y el nivel educativo (Ramírez y Núñez, 20I0). Durante las últimas décadas se ha experimentado un crecimiento preocupante en los casos de violencia en la pareja (Mohamed, Herrera y Carrañedo, 2014). Los estudios más recientes dirigen la mirada hacia una nueva tipología en la pareja que cada vez está más presente: la ejercida dentro de las parejas adolescentes (Cortés et al., 2014; Rodríguez, 20 I5; Valdivia y González, 20 I4). Las investigaciones han puesto de manifiesto que este fenómeno está muy presente en los jóvenes, constatándose la presencia de violencia en las relaciones de pareja como forma para resolver los conflictos (Pazos, Oliva y Hernando, 20l4). Otros autores, como, por ejemplo, Sebastián et al. (20l0), indican -en relación con la epidemiología de la violencia en las relaciones afectivas - que la violencia contra la pareja suele ocurrir más en jóvenes y adolescentes que en adultos, aunque de intensidad inferior.

Es importante destacar que la adolescencia es un periodo de transformación, exploración, autoanálisis y autoevaluación

(1)


que termina con la creación de un sentido coherente e integrado de uno mismo (Valdivia y González, 2014). Por tanto, se puede considerar que la adolescencia es una etapa clave para el aprendizaje, en la que las pautas de comportamiento que se adquieran van a influir en la formación tanto de los jóvenes como de los adultos. Asimismo, de acuerdo con Sebastián et al. (2010), la violencia en las relaciones afectivas entre jóvenes se produce en una fase en la que se empiezan a hacer realidad las primeras relaciones románticas anheladas $\mathrm{y}$, en muchos casos, soñadas, en las que varias de las pautas de interacción pueden dejar las puertas abiertas al abuso en las relaciones presentes y futuras. No hay que olvidar, como ya señalan otros autores (González-Ortega, Echeburúa y De Corral, 2008), que la violencia en las parejas jóvenes suele ser precursora de agresiones en parejas adultas. Por tanto, el estudio de las conductas violentas en las parejas juveniles es de gran relevancia, no solo por su alarmante tasa de prevalencia y sus consecuencias en la salud física y mental de las víctimas, sino por la etapa de la vida en la que se producen, una etapa que se caracteriza por ser el inicio de las relaciones románticas y en la que se aprenden las pautas de interacción en la pareja (Rodríguez, Riosvelasco y Castillo, 2018).

Los resultados de investigaciones en los últimos años en diferentes países muestran la alta prevalencia de la violencia entre los jóvenes (Cortés-Ayala et al., 20I4; MuñozRivas, Graña, O'Leary y González, 2007; Palmetto, Davidson, Breitbart y Rickert, 2013). En concreto, se ha encontrado que aproximadamente el $90 \%$ de los adolescentes encuestados, independientemente del sexo,admiten haber ejercido violencia verbal en contra de su pareja. Además, Cortés-Ayala et al. (2014) destacan que el $88 \%$ de los participantes de su investigación no perciben el maltrato por parte de su pareja sentimental; sin embargo, resaltan que una importante mayoría de jóvenes del estudio presentan maltrato técnico (i. e., no se perciben como maltratados, pero muestran evidencias de haber experimentado abuso en la relación).

La explicación de la violencia en la pareja es una cuestión que ha estado en constante debate. Concretamente, el debate sobre el género ha dado lugar a muchas equivocaciones, ideologizaciones y politizaciones, las cuales, más que arrojar luz con sus constructos, parecen producir más alarma y confusiones, de modo que afectan de lleno la teoría y la investigación científica dadas la diversidad $y$ las contradicciones en sus usos de interpretaciones (Rojas-Solís, 20I3).
Con respecto a esto, en un metaanálisis del 2014 (LópezCepero, Rodríguez, Rodríguez y Bringas, 2014), se analizó 1724 artículos sobre la violencia en las parejas. De estos, 159 artículos hicieron mención al género del participante, y de estos, 104 incluyeron solo a mujeres, 40 a ambos miembros y 15 solo a varones. En este sentido, se halló que casi en la totalidad de los estudios realizados se asignaban papeles de manera rígida a los hombres como agresores y a mujeres como víctimas. Solo algunos de los trabajos estudiaron a ambos géneros.

Por otra parte, las investigaciones han demostrado que la violencia predominante en la pareja es de tipo bidireccional. Un estudio llevado a cabo por Rodríguez (20I5), en el que se analizaron las respuestas de 740 alumnos (mujeres y varones), destacó que las tasas de violencia en la pareja ejercida por los jóvenes de diferente sexo son similares.Además, se observó una elevada proporción de bidireccionalidad en las conductas de agresión, siendo incluso superior la violencia física y psicológica de las mujeres sobre los hombres. Un 37,4\% en el caso de violencia física sobre un $17,9 \%$ que manifestaron ejercer los hombres, así como un $87,2 \%$ de mujeres frente a un $79,1 \%$ en hombres en la violencia psicológica. De igual forma, PazosGómez et al. (2014) realizaron un estudio con 712 sujetos (398 varones y 314 mujeres), cuyos resultados mostraron que ambos sexos eran ejecutores de la violencia en la pareja, señalando a las mujeres como más perpetradoras de la violencia física y verbal. De igual forma, otros estudios enfocados en el estudio de violencia en parejas universitarias (Celis-Sauce y Rojas, 2015; Fernández-González, O'Leary y Muñoz-Rivas, 20 I4; Palmetto et al., 20I3;Viejo, 20I4; Volpe, Morales-Alemás y Teitelman, 20I4), destacan que la violencia que se produce en estas relaciones, la mayoría de las veces, es de tipo bidireccional.

En atención a los tres tipos de violencia típicos de este fenómeno (física, psicológica y sexual), los estudios muestran que ambos sexos se ven inmersos en la violencia en ambos roles, como víctimas y como agresores. Asimismo, los estudios indican que la violencia verbal emocional era el subtipo de agresión más frecuente entre las parejas (Pazos et al., 20l4), con independencia del sexo. De acuerdo con Lazarevich, Irigoyen, Sokolova y Delgadillo (20/2), en un estudio que realizaron con 729 estudiantes, informaron de una alta prevalencia de violencia verbal emocional (75\%). Por otra parte, Viejo (20I4) analizó la implicación en la violencia grave y leve tanto de chicos como de chicas, y halló resultados similares en ambos.

En la misma línea, en una investigación en la que se analizan aquellas creencias y actitudes sobre los roles de género 
en jóvenes estudiantes con el fin de comprobar si existen diferencias en función del nivel de estudio y del sexo, se obtuvo que las chicas presentaban más actitudes sexistas relacionadas con el contexto laboral y más actitudes trascendentes en el ámbito social; mientras que en los chicos los resultados no fueron significativos. De lo anterior se desprende que, en España, aumenta de manera progresiva el número de jóvenes que sufren algún tipo de abuso por parte de sus parejas (Muñoz y Echeburúa 2015).

En relación con la victimización, un estudio en México que analizó la relación entre el apoyo social percibido de familiares y amigos y la victimización en relaciones de noviazgo encontró que los hombres experimentan una frecuencia mayor de violencia con relación a las mujeres, y que la percepción de apoyo de las amistades se asoció de forma inversa con varios tipos de victimización. Por el contrario, en las mujeres el apoyo social de ambas fuentes tuvo poca relación con la violencia experimentada (Rodríguez et al., 2018).

La violencia en las relaciones de pareja adolescente representa un grave problema social del que están haciendo eco las instituciones legislativas, sanitarias y educativas (Sebastián et al., 2010), el cual conlleva importantes consecuencias a corto y largo plazo en la salud de las víctimas (Muñoz-Rivas, Gámez-Guadix, Graña y Fernández, 20l0). Los estudios confirman consecuencias en la salud física y psicológica de los adolescentes tales como el abuso de sustancias, conductas sexuales de riesgo, bajo rendimiento académico, abandono de los estudios y baja autoestima. Además, existen estudios que informan de una relación con otro tipo de comportamientos preocupantes, como, por ejemplo, suicidios, embarazos no deseados, trastornos alimentarios, aumento del sentimiento de culpa, ira, dolor, ansiedad y depresión (Martínez,Vargas y Novoa, 2016). Por su parte, Sebastián et al. (2010) recalcan la importancia de estas consecuencias o repercusiones de la violencia (física, psicológica y sexual) en la vida de las personas, y subrayan la urgente necesidad de realizar un esfuerzo para erradicarla, detectarla y poner medios para prevenirla. Al mismo tiempo, hacen notar cómo los fallos y el desconocimiento que tienen hoy en día los jóvenes sobre lo que implica una relación de pareja se relaciona con un escaso aprendizaje emocional, algo que se atribuye a que no se enseña a los jóvenes cómo comportarse en este campo.

En conformidad con las elevadas cifras y sus consecuencias en esta población, es importante contar con datos que permitan el estudio de este fenómeno y contribuyan a la comunidad científica para entender que la violencia de pareja en jóvenes, tanto de mujeres hacia hombres como viceversa, es un grave problema de salud pública. Se hace necesario estudiar el fenómeno de manera exhaustiva a fin de estar en capacidad de ayudar al diseño de campañas reales de prevención y de protección, pues el problema se puede agravar en sus relaciones futuras.

El objetivo general de esta investigación es analizar la relación entre la perpetración de la violencia de pareja con la victimización de dicha violencia en una muestra de estudiantes universitarios.

Los objetivos específicos son los siguientes: (I) conocer la frecuencia de agresiones cometidas y recibidas; (2) conocer la frecuencia de la violencia sexual en función del género; (3) conocer la frecuencia de la violencia psicológica en función del género; (4) estudiar cómo se distribuye la variable celos en función del género.

\section{Método}

\section{Participantes}

En el estudio participaron inicialmente 160 jóvenes universitarios de manera anónima y voluntaria. De los 160 cuestionarios recogidos se descartaron 23 (14,37\%), por alguna de las siguientes razones: (a) encontrarse incompletos, (b) tener una elevada cantidad de datos perdidos o (c) no cumplir los criterios de inclusión (tener o haber tenido una relación sentimental y encontrarse en una edad entre los 18 y 25 años). Finalmente, se consideró en el estudio la participación de 137 estudiantes ( 101 mujeres, 36 hombres) de edades comprendidas entre los 18 y los 25 años $(M=19,8, D T=2,17)$, $y$ de universidades de la Comunidad de Madrid. En concreto, de las Facultades de Psicología y Criminología de las Universidad Autónoma de Madrid y Universidad CEU San Pablo, respectivamente.

\section{Diseño, variables e instrumentos}

Se utilizó un diseño factorial intersujetos con el género (mujer, hombre), así como la prevalencia, la perpetración y la victimización como variables independientes.

Las variables dependientes fueron los tres tipos de violencia de género comúnmente estudiados en las investigaciones (física, psicológica y sexual), más las dimensiones celos y control.

En el marco de esta investigación se entiende por prevalencia la medida de frecuencia de las conductas violentas, 
Tabla I. Características sociodemográficas de los participantes en función del sexo $(n=|4|)$

\begin{tabular}{|c|c|c|}
\hline \multicolumn{2}{|c|}{ Variable } & Muestra de análisis \\
\hline \multicolumn{2}{|c|}{ N. ${ }^{\circ}$ de jóvenes seleccionados } & 137 \\
\hline \multirow{2}{*}{ Género } & Mujeres & 101 \\
\hline & Hombres & 36 \\
\hline \multirow{8}{*}{ Edad } & 18 & 59 \\
\hline & 19 & 27 \\
\hline & 20 & 6 \\
\hline & 21 & 9 \\
\hline & 22 & 12 \\
\hline & 23 & 14 \\
\hline & 24 & 3 \\
\hline & 25 & 6 \\
\hline \multirow{3}{*}{ Nacionalidad } & Español & 127 \\
\hline & Extranjero & 4 \\
\hline & Doble Nacionalidad & 2 \\
\hline \multirow{4}{*}{ Composición familiar } & Completa & 106 \\
\hline & Monoparental & 19 \\
\hline & Otros & 5 \\
\hline & Solo & 5 \\
\hline \multirow{2}{*}{ Trabajo } & No & 104 \\
\hline & Sí & 33 \\
\hline \multirow{4}{*}{ Estado Socioeconómico } & Alto & 3 \\
\hline & Medio-alto & 37 \\
\hline & Medio & 79 \\
\hline & Medio-bajo & II \\
\hline \multirow{4}{*}{ Orientación Sexual } & Heterosexual & 124 \\
\hline & Homosexual & I \\
\hline & Bisexual & 8 \\
\hline & No estoy seguro & 3 \\
\hline
\end{tabular}

es decir, el número de casos que los hechos objeto de este estudio aparecen en el grupo de población; por perpetración se hace referencia a quien ejecuta un acto violento en contraposición a la víctima que es la que recibe los actos violentos (victimización).
A fin de determinar la prevalencia, la perpetración y la victimización de la violencia en jóvenes que mantienen una relación sentimental y de pareja se utilizó un cuestionario elaborado ad hoc basado en la investigación "Partners and peers: sexual and dating violence amongst NYC youth" (Fry, O'Connor, Davidson, Paz, Palmetto y Rickert, 2008), realizada para determinar la prevalencia y la perpetración de la violencia en jóvenes en las relaciones sexuales o sentimentales, con algunas pequeñas variaciones, $y$ obviando la parte dedicada a la salud por no ser objeto de estudio en la investigación. El cuestionario, con índice de consistencia interna de ,78, estaba compuesto por 118 ítems y cuatro preguntas cortas, divididas en seis secciones, las cuales se organizan tal como se muestra en la tabla 2.

El análisis objeto de este estudio se centró, principalmente, en la Sección I y 3 del cuestionario, que hacen mención a los datos sociodemográficos y al tipo de violencia existente.

\section{Procedimiento}

Se solicitó a los participantes su colaboración, así como se les informó de la anonimidad del cuestionario. Se suministró de forma colectiva con una duración de aplicación de 35 minutos. Los participantes fueron informados de: (I) el procedimiento de la investigación y las posibles dificultades; (2) la posibilidad de no aceptar la participación en el estudio; (3) el anonimato y la privacidad de los datos.

A fin de llevar a cabo los distintos análisis se utilizó el paquete informático SPSS (Stadistical Package for Social Sciences) versión 20.0 para Windows, por el cual se examinó el análisis de frecuencias con el propósito de describir las conductas de violencia que reportaron los jóvenes en las relaciones de pareja. Además, se estudió por separado al grupo de mujeres del de hombres a fin de

Tabla 2. Ítems de estudio

Sección I Contiene ítems relacionados con las características sociodemográficas de los sujetos e historia de violencia durante la infancia (n ${ }^{\circ}$ de ítems $=15$ )

Sección 2 Ítems sobre las relaciones y vida sexual, de ahora y en el pasado $\left(\mathrm{n}^{\circ}{ }^{\circ}\right.$ de ítems $\left.=28\right)$

Sección 3 Ítems para determinar la respuesta a situaciones tanto propias como a la pareja $\left(n .^{\circ}\right.$ de ítems $\left.=43\right)$, en los que se estudia el tipo de violencia existente

Sección 4 Ítems centrados en la victimización, es decir, con el objetivo de determinar el grado de violencia que ha sufrido el sujeto en las relaciones sentimentales que ha tenido $\left(n .^{\circ}\right.$ de ítems $\left.=23\right)$

Sección 5 Ítems relacionados con la violencia sexual que haya podido sufrir el sujeto. Violencia procedente de alguien diferente de la pareja (n. ${ }^{\circ}$ de ítems $=5$ )

Sección 6 Ítems relacionados con la forma de actuar de la persona frente a situaciones de violencia propia o ajena, la percepción de esta sobre la violencia, así como la información que tiene acerca de esta $\left(n{ }^{\circ}\right.$ de ítems $\left.=4\right)$ 
analizar si existían diferencias significativas en ambos grupos, calculándose los porcentajes, las medias y el DT de los comportamientos mostrados en la relación.Asimismo, se realizó la prueba de $U$ Mann Whitney con la finalidad de determinar si existían diferencias significativas entre el grupo de mujeres $y$ hombres en determinadas variables.

\section{Resultados}

Los datos descriptivos de las agresiones cometidas y sufridas (tabla 3) muestran mayor frecuencia de agresiones de tipo psicológico con independencia del género. También se evidencia la existencia de comportamientos de celos y control, aunque en menor medida que los de violencia psicológica.

Con respecto a la diferenciación del grupo de hombres del de mujeres se encontró que no existían prácticamente diferencias significativas entre ambos grupos, siendo tanto las mujeres como los hombres agresores y víctimas en estas relaciones.

Los datos informan que no existe una prevalencia elevada en agresiones físicas y sexuales. La mayoría de los jóvenes de la muestra indicaron no haber realizado ni sufrido nunca ese comportamiento hacia o por parte de su pareja. Por el contrario, en cuanto a las agresiones de carácter psicológico/emocional, así como las conductas de celos y control eran más frecuentes en este tipo de relaciones. En el caso de violencia psicológica se registraron más agresiones sufridas que ejercidas.

Tabla 3. Medidas generales en frecuencia de las agresiones cometidas $y$ sufridas

\begin{tabular}{ccccccccc} 
Agresión & Subescala & N & Ítems & Mínimo & Máximo & M & DT \\
\hline \multirow{2}{*}{ Psicológica } & Cometida & 137 & 12 & II & 24 & 15,12 & 3,19 \\
& Sufrida & 137 & 12 & II & 38 & 18,86 & 4,57 \\
\hline \multirow{2}{*}{ Física } & Cometida & 137 & 4 & 4 & 7 & 4,17 &, 54 \\
& Sufrida & 137 & 4 & 2 & 10 & 4,15 &, 74 \\
\hline \multirow{2}{*}{ Sexual } & Cometida & 137 & 4 & 3 & 8 & 4,45 &, 87 \\
& Sufrida & 137 & 4 & 4 & 12 & 4,63 & 1,21 \\
\multirow{2}{*}{ Celos } & Cometida & 137 & 4 & 4 & 12 & 5,36 & 1,68 \\
& Sufrida & 137 & 4 & 1 & 13 & 5,49 & 2,13 \\
\hline \multirow{2}{*}{ Control } & Cometida & 137 & 5 & 4 & 16 & 5,85 & 1,66 \\
& Sufrida & 137 & 5 & 0 & 11 & 5,58 & 1,56 \\
\hline
\end{tabular}

$\mathrm{Al}$ analizar las subescalas de violencia física y los ítems que la componen (tabla 4) se puede destacar que la mayoría de los jóvenes contestaron que nunca habían ejercido o sufrido este tipo de comportamientos, de manera que los resultados del grupo de las mujeres y de los hombres fueron muy similares con apenas diferencias entre ambos grupos.

Con respecto a las agresiones de carácter sexual (tabla 5 ), de los cuatro ítems que evaluaban este comportamiento solo uno de ellos (ítem 4) destacó "la besé cuando ella no quería" o "me besó cuando yo no quería"; los resultados muestran que un $28,28 \%$ declaran haber ejercido este comportamiento, siendo ligeramente superior en hombres $(4 I, 67 \%)$ que en mujeres $(22,77 \%)$. Con respecto al comportamiento sufrido los datos informan que no existen diferencias con respecto a las mujeres y a los hombres con un $29,7 \%$ y un $33,33 \%$, respectivamente. No obstante, de los que mostraron haber ejercido dicho comportamiento, una gran parte de las respuestas se encontró en las opciones de "pocas veces".

En cuanto a las agresiones de carácter psicológico, puede observarse un aumento en la frecuencia general. Los ítems 3 y 5 son los que mostraron más variabilidad en las respuestas (tabla 6). Con respecto a la perpetración de agresiones de carácter psicológico, a pesar de mostrar resultados parecidos las mujeres reportaron haber ejercido más este tipo de comportamientos.

A continuación, se estudió el grado de perpetración de conductas violentas. Los ítems con mayor número de respuestas declaradas por los jóvenes fueron: (a) "he hecho algo para que se sienta celoso", el 50,49\% de las mujeres y el $52,78 \%$ de los hombres informaron de haber realizado este comportamiento; (b) "recordar algo malo que hizo en el pasado", el $75,24 \%$ de las mujeres y el $55,56 \%$ de los hombres; (c) "he dicho cosas para enfadarle", el 50,5\% mujeres y el 47,22\% de los hombres; (d) "hablar de manera hostil o con mal tono de voz"; el 69,31\% en mujeres y el $58,33 \%$ en hombres; (e) "culpar de los problemas", el $60,4 \%$ de las mujeres y el $55,6 \%$ de los hombres informaron del comportamiento. No obstante, en cuanto a frecuencia de este comportamiento, en la mayoría de los casos la respuesta que se encontró fue "pocas veces".

Por otra parte, en relación con la victimización (haber sufrido el comportamiento), tanto hombres como mujeres informaron haber sufrido este comportamiento. Sin embargo, las mujeres informaron haber sufrido más este comportamiento por parte de sus parejas en los ítems 3 y 5 . 
Tabla 4. Puntajes medios de las subescalas y los ítems que componen la escala de violencia física

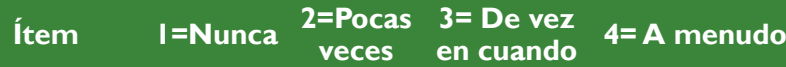

Agresiones físicas cometidas

\begin{tabular}{|c|c|c|c|c|}
\hline $\begin{array}{l}\text { (I) Le he } \\
\text { lanzado } \\
\text { algo a mi } \\
\text { pareja }\end{array}$ & $97,81 \%$ & $2,19 \%$ & $0 \%$ & $0 \%$ \\
\hline Mujeres & $97,03 \%$ & $2,97 \%$ & $0 \%$ & $0 \%$ \\
\hline Hombres & $100 \%$ & $0 \%$ & $0 \%$ & $0 \%$ \\
\hline $\begin{array}{l}\text { (2) Le he } \\
\text { golpeado }\end{array}$ & $98,54 \%$ & ,73\% & ,73\% & $0 \%$ \\
\hline Mujeres & $99,01 \%$ & $0 \%$ & ,99\% & $0 \%$ \\
\hline Hombres & $97,22 \%$ & $2,78 \%$ & $0 \%$ & $0 \%$ \\
\hline $\begin{array}{l}\text { (3) Le he } \\
\text { empujado }\end{array}$ & $91,97 \%$ & $6,57 \%$ & ,73\% & ,73\% \\
\hline Mujeres & $89,11 \%$ & $8,91 \%$ & ,99\% & ,99\% \\
\hline Hombres & $100 \%$ & $0 \%$ & $0 \%$ & $0 \%$ \\
\hline $\begin{array}{l}\text { (4) Le he } \\
\text { abofeteado } \\
\text { o tirado } \\
\text { del pelo }\end{array}$ & $98,54 \%$ & ,73\% & ,73\% & $0 \%$ \\
\hline Mujeres & $99,01 \%$ & ,99\% & $0 \%$ & $0 \%$ \\
\hline Hombres & $97,22 \%$ & $0 \%$ & $2,78 \%$ & $0 \%$ \\
\hline
\end{tabular}

Agresiones físicas sufridas

\begin{tabular}{|c|c|c|c|c|}
\hline $\begin{array}{l}\text { (I) Me ha } \\
\text { lanzado } \\
\text { algo mi } \\
\text { pareja }\end{array}$ & $96,35 \%$ & $2,19 \%$ & I,46\% & $0 \%$ \\
\hline Mujeres & $98,02 \%$ & I,98\% & $0 \%$ & $0 \%$ \\
\hline Hombres & $91,65 \%$ & $2,78 \%$ & $5,56 \%$ & $0 \%$ \\
\hline $\begin{array}{l}\text { (2) Me ha } \\
\text { golpeado }\end{array}$ & $97,81 \%$ & $1,46 \%$ & ,73\% & $0 \%$ \\
\hline Mujeres & $99,01 \%$ & ,99\% & $0 \%$ & $0 \%$ \\
\hline Hombres & $94,44 \%$ & $2,78 \%$ & $2,78 \%$ & $0 \%$ \\
\hline $\begin{array}{l}\text { (3) Me ha } \\
\text { empujado }\end{array}$ & $93,43 \%$ & $2,92 \%$ & $2,19 \%$ & $0 \%$ \\
\hline Mujeres & $95,05 \%$ & $3,94 \%$ & ,99\% & $0 \%$ \\
\hline Hombres & $88,89 \%$ & $0 \%$ & $5,56 \%$ & $0 \%$ \\
\hline $\begin{array}{l}\text { (4) Me ha } \\
\text { abofeteado } \\
\text { o tirado } \\
\text { del pelo }\end{array}$ & $96,35 \%$ & $2,19 \%$ & $0 \%$ & $0 \%$ \\
\hline Mujeres & $99,01 \%$ & ,99\% & $0 \%$ & $0 \%$ \\
\hline Hombres & $88,89 \%$ & $5,56 \%$ & $0 \%$ & $0 \%$ \\
\hline
\end{tabular}

Por último, se analizaron las conductas de celos y control en la relación (tabla 7). Se encontraron datos similares tanto para hombres como para mujeres. En relación con los celos, tanto en la victimización como en la perpetración, destacaron los ítems 2 (acusar de flirtear con otra
Tabla 5. Puntajes medios de las subescalas y los ítems que componen la escala de violencia sexual

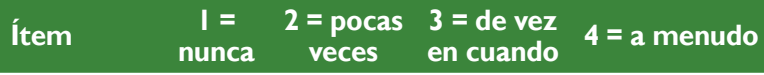

Agresiones sexuales cometidas

\begin{tabular}{rrccc}
$\begin{array}{l}\text { (I) He tocado } \\
\text { sexualmente } \\
\text { a mi pareja } \\
\text { cuando esta } \\
\text { no quería }\end{array}$ & $92,7 \%$ & $5,11 \%$ & $1,46 \%$ & $0 \%$ \\
\hline Mujeres & $94,06 \%$ & $4,95 \%$ & $0 \%$ & $0 \%$ \\
Hombres & $88,89 \%$ & $5,56 \%$ & $5,56 \%$ & $0 \%$
\end{tabular}

\begin{tabular}{|c|c|c|c|c|}
\hline $\begin{array}{l}\text { (2) La he forzado } \\
\text { a tener sexo } \\
\text { cuando esta } \\
\text { no quería }\end{array}$ & $98,54 \%$ & $0 \%$ & ,73\% & $0 \%$ \\
\hline Mujeres & $98,02 \%$ & $0 \%$ & ,99\% & $0 \%$ \\
\hline Hombres & $100 \%$ & $0 \%$ & $0 \%$ & $0 \%$ \\
\hline $\begin{array}{l}\text { (3) Le he amenazado } \\
\text { para intentar } \\
\text { tener sexo con } \\
\text { él/ella }\end{array}$ & $100 \%$ & $0 \%$ & $0 \%$ & $0 \%$ \\
\hline Mujeres & $100 \%$ & $0 \%$ & $0 \%$ & $0 \%$ \\
\hline Hombres & $100 \%$ & $0 \%$ & $0 \%$ & $0 \%$ \\
\hline $\begin{array}{l}\text { (4) Le bese cuando } \\
\text { él/ella no quería }\end{array}$ & $72,26 \%$ & $21,17 \%$ & $5,11 \%$ & I,46\% \\
\hline Mujeres & $77,23 \%$ & $18,81 \%$ & $2,97 \%$ & ,99\% \\
\hline Hombres & $58,33 \%$ & $27,78 \%$ & $|I| I \%$, & $2,78 \%$ \\
\hline
\end{tabular}

Agresiones sexuales sufridas

(I) Mi pareja me ha tocado sexualmente cuando yo no quería $\begin{array}{llll}87,59 \% & 9,49 \% & 2,92 \% & 0 \%\end{array}$ que lo hiciese

$\begin{array}{ccccc}\text { Mujeres } & 87,13 \% & 10,89 \% & 1,98 \% & 0 \% \\ \text { Hombres } & 88,89 \% & 5,56 \% & 5,56 \% & 0 \%\end{array}$

(2) Me ha forzado a tener sexo cuando yo no quería

$97,08 \% \quad 2,19 \% \quad, 73 \% \quad 0 \%$

$\begin{array}{rrrrr}\text { Mujeres } & 97,03 \% & 1,98 \% & , 99 \% & 0 \% \\ \text { Hombres } & 97,22 \% & 2,78 \% & 0 \% & 0 \%\end{array}$

(3) Él/ella me ha amenazado para intentar tener $98,54 \% \quad, 73 \% \quad 0 \%$ , $73 \%$ sexo conmigo

$\begin{array}{ccccc}\text { Mujeres } & 99,01 \% & 0 \% & 0 \% & , 99 \% \\ \text { Hombres } & 97,22 \% & 2,78 \% & 0 \% & 0 \%\end{array}$

(4) Él/ella me $\begin{array}{lllll}\text { beso cuando } & 69,34 \% & 22,63 \% & 5,84 \% & 2,19 \%\end{array}$ yo no quería

\begin{tabular}{lllll} 
Mujeres & $70,30 \%$ & $22,77 \%$ & $4,95 \%$ & $1,98 \%$ \\
Hombres & $66,77 \%$ & $22,22 \%$ & $8,33 \%$ & $2,78 \%$ \\
\hline
\end{tabular}


Tabla 6. Puntajes medios de las subescalas y los ítems que componen la escala de violencia psicológica

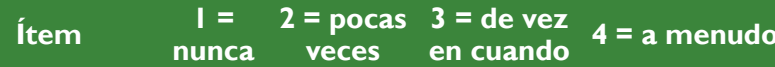

Agresiones psicológicas cometidas

(I) He hecho algo

$\begin{array}{rcccc}\begin{array}{l}\text { para que él/ } \\ \text { ella se sienta } \\ \text { celoso }\end{array} & 48,91 \% & 37,96 \% & 11,68 \% & 1,46 \% \\ \text { Mujeres } & 49,5 \% & 36,63 \% & 11,88 \% & 1,98 \% \\ \text { Hombres } & 47,22 \% & 41,67 \% & 11,11 \% & 0 \%\end{array}$

(2) He destrozado

o amenazado

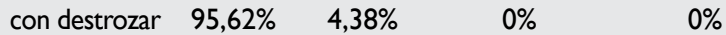

algo que $\mathrm{mi}$

pareja valora

Hombres $94,44 \%$

$3,96 \% \quad 0 \% \quad 0 \%$

(3) Le he recorda-

$\begin{array}{lllll}\text { do algo malo } & 29,93 \% & 32,85 \% & 24,82 \% & 12,42 \%\end{array}$

$32,85 \%$

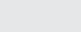

$15,84 \%$

Mujeres $24,75 \% \quad 33,66 \%$

$25,74 \% \quad 15,84 \%$

Hombres 44,44\%

$30,56 \%$

$22,22 \%$

$2,78 \%$

(4) He dicho cosas

$\begin{array}{lllll}\text { para enfadarle } & 50,36 \% & 37,23 \% & 10,95 \% & \mathrm{I}, 46 \%\end{array}$

a él/ella

Mujeres $49,5 \%$

$39,6 \%$

$9,9 \%$

,99\%

Hombres 52,78\%

$30,56 \%$

$13,89 \%$

$2,78 \%$

(5) Le he hablado

de manera

hostil o con $\quad 33,58 \% \quad 45,26 \% \quad 14,6 \% \quad 5,84 \%$

mal tono

de voz

$\begin{array}{rrrcr}\text { Mujeres } & 30,69 \% & 44,55 \% & 16,83 \% & 6,93 \% \\ \text { Hombres } & 41,67 \% & 47,22 \% & 8,33 \% & 2,78 \%\end{array}$

(6) Le he

$\begin{array}{llll}\text { insultado con } 91,97 \% & 7,3 \% & 0 \% & 0 \%\end{array}$

humillaciones

$\begin{array}{rrrrr}\text { Mujeres } & 93,07 \% & 5,94 \% & 0 \% & 0 \% \\ \text { Hombres } & 88,89 \% & \text { II,1 } 1 \% & 0 \% & 0 \%\end{array}$

(7) Le he ridiculi-

zado o me he

$\begin{array}{lllll}\text { reído de él/ } & 94,16 \% & 4,38 \% & \mathrm{I}, 46 \% & 0 \%\end{array}$

ella delante

de otros

$\begin{array}{llll}\text { Mujeres } 93,07 \% & 4,95 \% & 1,98 \% & 0 \%\end{array}$

Hombres $97,22 \% \quad 2,78 \% \quad 0 \% \quad 0 \%$

(8) Le he culpado

a él/ella de los $\quad 44,53 \% \quad 37,23 \% \quad 17,52 \% \quad$,73\%

problemas

$\begin{array}{rrrrr}\text { Mujeres } & 39,6 \% & 38,61 \% & 0 \% & , 99 \% \\ \text { Hombres } & 44,53 \% & 37,23 \% & 0 \% & , 73 \%\end{array}$

(9) He intentado

$\begin{array}{lllll}\text { delibera- } & 91,24 \% & 8,76 \% & 0 \% & 0 \%\end{array}$

asustarle/la

\begin{tabular}{rcccc} 
Mujeres & $89,11 \%$ & $10,89 \%$ & $8,03 \%$ & $0 \%$ \\
Hombres & $97,22 \%$ & $2,78 \%$ & $8,91 \%$ & $0 \%$ \\
\hline
\end{tabular}

(10) Le ignoró o

$\begin{array}{llll}\text { le trató con } 75,91 \% & 14,6 \% & 5,56 \% & 0,73 \%\end{array}$

indiferencia

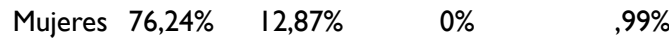

$\begin{array}{llll}\text { Hombres } & 75 \% & 19,44 \% & 0 \%\end{array}$

(II) He amenaza-

do a mi

$\begin{array}{lllll}\text { pareja con } & 97,08 \% & 2,92 \% & 0 \% & 0 \%\end{array}$

pegarle 0

lanzarle cosa

$\begin{array}{ccccc}\text { Mujeres } & 96,04 \% & 3.96 \% & 0 \% & 0 \% \\ \text { Hombres } & 100 \% & 0 \% & 0 \% & 0 \%\end{array}$

(12) $\mathrm{He}$

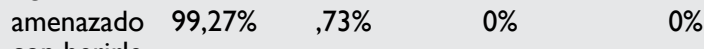

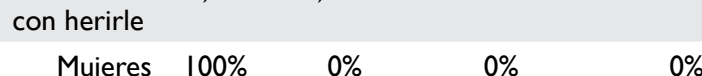

Hombres $97,22 \% \quad 2,78 \% \quad 0 \% \quad 0 \%$

\section{Agresiones psicológicas sufridas}

(I) Él/ella ha hecho

$\begin{array}{lllll}\text { algo para que } & 51,09 \% & 29,93 \% & 12,41 \% & 4,38 \%\end{array}$

yo me sienta

celoso

$\begin{array}{rcccc}\text { Mujeres } & 51,49 \% & 34,65 \% & 8,91 \% & 3,96 \% \\ \text { Hombres } & 50 \% & 16,67 \% & 22,22 \% & 5,56 \%\end{array}$

(2) Él/ella ha

amenazado

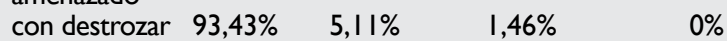

algo que yo

valoro

$\begin{array}{rrccc}\text { Mujeres } & 95,05 \% & 2,97 \% & 1,98 \% & 0 \% \\ \text { Hombres } & 88,89 \% & 11,11 \% & 0 \% & 0 \%\end{array}$

(3) Me ha recordado

$\begin{array}{lllll}\text { algo que hice } \quad 33,58 \% & 35,04 \% & 23,36 \% & 8,03 \%\end{array}$

mal en el pasado

Mujeres $29,7 \%$

$37,62 \%$

$25,74 \%$

$6,93 \%$

Hombres 44,44\%

$27,78 \%$

$16,67 \%$

$1 \mathrm{I}, \mathrm{II} \%$

(4) Me ha dicho

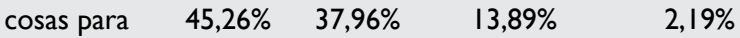
enfadarme

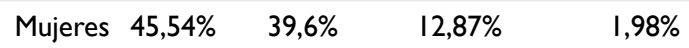

Hombres $44,44 \% \quad 33,33 \% \quad 16,67 \% \quad 2,78 \%$

(5) Me ha hablado

de manera

$\begin{array}{llll}\text { hostil o con } \quad 32,86 \% & 40,15 \% & 19,71 \% & 6,57 \%\end{array}$

mal tono de

voz

$\begin{array}{rrrrr}\text { Mujeres } & 29,7 \% & 43,56 \% & 19,8 \% & 5,94 \% \\ \text { Hombres } & 41,67 \% & 30,56 \% & 19,44 \% & 8,33 \%\end{array}$

(6) Me ha

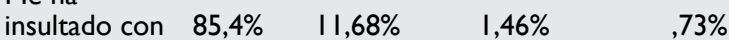

humillaciones

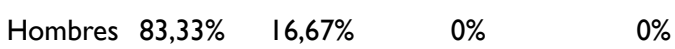

(7) Me ha ridiculizado

$\begin{array}{lllll}\begin{array}{l}\text { o se ha rédo de } \\ \text { mi delante de }\end{array} & 88,32 \% & 9,49 \% & 1,46 \% & , 73 \%\end{array}$

otros

$\begin{array}{lllll}\text { Mujeres } & 86,42 \% & 11,88 \% & , 99 \% & , 99 \%\end{array}$

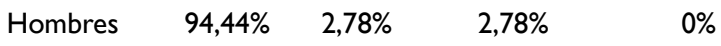




\begin{tabular}{|c|c|c|c|c|}
\hline $\begin{array}{l}\text { (8) Me ha culpado } \\
\text { de los prob- } \\
\text { lemas }\end{array}$ & $45,26 \%$ & $38,69 \%$ & $13,14 \%$ & $2,92 \%$ \\
\hline Mujeres & $43,56 \%$ & $38,61 \%$ & I5,84\% & I,98\% \\
\hline Hombres & $50 \%$ & $38,89 \%$ & $5,56 \%$ & $5,56 \%$ \\
\hline $\begin{array}{l}\text { (9) Me ha intenta- } \\
\text { do deliberada- } \\
\text { mente asustar }\end{array}$ & $93,43 \%$ & $2,92 \%$ & $2,19 \%$ & $0 \%$ \\
\hline Mujeres & $93,07 \%$ & $3,96 \%$ & $2,97 \%$ & $0 \%$ \\
\hline Hombres & $94,44 \%$ & $0 \%$ & $0 \%$ & $0 \%$ \\
\hline $\begin{array}{l}\text { (10) Me ignora o } \\
\text { me trata con } \\
\text { indiferencia }\end{array}$ & $72,99 \%$ & $16,79 \%$ & $6,57 \%$ & ,73\% \\
\hline Mujeres & $75,25 \%$ & $17,82 \%$ & $5,94 \%$ & $0 \%$ \\
\hline Hombres & $66,67 \%$ & I3,89\% & $8,33 \%$ & $2,78 \%$ \\
\hline $\begin{array}{l}\text { (II) Mi pareja me } \\
\text { ha amenazado } \\
\text { con pegarme } \\
\text { o lanzarme } \\
\text { cosas }\end{array}$ & $97,08 \%$ & $2,19 \%$ & ,73\% & $0 \%$ \\
\hline Mujeres & $99,01 \%$ & ,99\% & $0 \%$ & $0 \%$ \\
\hline Hombres & $91,67 \%$ & $5,56 \%$ & $2,78 \%$ & $0 \%$ \\
\hline $\begin{array}{l}\text { (12) Me ha } \\
\text { amenazado } \\
\text { con herirme }\end{array}$ & $95,62 \%$ & $3,65 \%$ & ,73\% & $0 \%$ \\
\hline Mujeres & $96,04 \%$ & $2,97 \%$ & ,99\% & $0 \%$ \\
\hline Hombres & $94,44 \%$ & $5,56 \%$ & $0 \%$ & $0 \%$ \\
\hline
\end{tabular}

persona) y 3 (enfadarse por hablar con otro chico o chica). Con respecto a los comportamientos de control y su relación con la perpetración y victimización (tabla 8) se destacan los ítems 3 (saber dónde está en todo momento) y 4 (mirar el teléfono); en este caso los hombres informaron haber ejercido y sufrido más este tipo de comportamientos.

Como últimos análisis realizados se consideró interesante comprobar si existían diferencias significativas entre el grupo de hombres y el grupo de mujeres en los distintos comportamientos objeto de estudio, para lo cual se realizó un análisis no paramétrico del comportamiento de ambos grupos de estudio con relación a las variables de análisis.

De todas las variables analizadas solo se hallaron diferencias significativas entre ambos grupos en las conductas de acoso $(p<0,05)$ y las agresiones sexuales $(p<0,05)$.

\section{Discusión}

Este artículo examinó la prevalencia de las principales conductas violentas (recibidas y ejercidas) en las relaciones de pareja, físicas, sexuales y psicológicas. Se examinaron también las conductas de celos y de control asociadas a la relación.
Tabla 7. Comportamientos relacionados con los celos

\begin{tabular}{|c|c|c|c|c|}
\hline Ítem & $\begin{array}{c}I= \\
\text { nunca }\end{array}$ & $\begin{array}{c}2= \\
\text { pocas } \\
\text { veces }\end{array}$ & $\begin{array}{l}3=\text { de } \\
\text { vez en } \\
\text { cuando }\end{array}$ & $\begin{array}{c}4=\mathrm{a} \\
\text { menudo }\end{array}$ \\
\hline \multicolumn{5}{|c|}{ Comportamientos hacia la pareja } \\
\hline $\begin{array}{l}\text { (I) Le seguí para ver con quién } \\
\text { estaba y con quien iba }\end{array}$ & $96,35 \%$ & $3,65 \%$ & $0 \%$ & $0 \%$ \\
\hline Mujeres & $96,04 \%$ & $3,96 \%$ & $0 \%$ & $0 \%$ \\
\hline Hombres & $97,22 \%$ & $2,78 \%$ & $0 \%$ & $0 \%$ \\
\hline $\begin{array}{l}\text { (2) Le he acusado de flirtear } \\
\text { con otras personas }\end{array}$ & $60,58 \%$ & $33,58 \%$ & $4,38 \%$ & $\mathrm{I}, 46 \%$ \\
\hline Mujeres & $61,39 \%$ & $32,67 \%$ & $3,96 \%$ & $1,98 \%$ \\
\hline Hombres & $58,33 \%$ & $36,11 \%$ & $5,56 \%$ & $0 \%$ \\
\hline $\begin{array}{l}\text { (3) Me enfado cuando habla } \\
\text { con otro chico/a }\end{array}$ & $59,12 \%$ & $32,12 \%$ & $4,38 \%$ & $4,38 \%$ \\
\hline Mujeres & $59,41 \%$ & $32,67 \%$ & $2,97 \%$ & $4,95 \%$ \\
\hline Hombres & $58,33 \%$ & $30,36 \%$ & $8,33 \%$ & $2,78 \%$ \\
\hline $\begin{array}{l}\text { (4) Sospecho muchas veces } \\
\text { que me es infiel }\end{array}$ & $79,56 \%$ & $12,4 \mid \%$ & $4,38 \%$ & $3,65 \%$ \\
\hline Mujeres & $80,20 \%$ & $12,87 \%$ & $1,98 \%$ & $4,95 \%$ \\
\hline Hombres & $77,78 \%$ & $11,11 \%$ & $11,11 \%$ & $0 \%$ \\
\hline \multicolumn{5}{|c|}{ Comportamientos de la pareja } \\
\hline $\begin{array}{l}\text { (I) Me siguió para ver con } \\
\text { quién estaba y con quién iba }\end{array}$ & $94,89 \%$ & $3,65 \%$ & ,73\% & ,73\% \\
\hline Mujeres & $96,04 \%$ & $1,98 \%$ & ,99\% & ,99\% \\
\hline Hombres & $91,67 \%$ & $8,33 \%$ & $0 \%$ & $0 \%$ \\
\hline $\begin{array}{l}\text { (2) Me acusó de flirtear con } \\
\text { otras personas }\end{array}$ & $54,01 \%$ & $25,55 \%$ & $13,87 \%$ & $5,11 \%$ \\
\hline Mujeres & $53,47 \%$ & $24,75 \%$ & $16,83 \%$ & $4,95 \%$ \\
\hline Hombres & $55,56 \%$ & $27,78 \%$ & $5,56 \%$ & $5,56 \%$ \\
\hline $\begin{array}{l}\text { (3) Se enfada cuando hablo } \\
\text { con otro chico/a }\end{array}$ & $56,2 \%$ & $32,12 \%$ & $6,57 \%$ & $2,19 \%$ \\
\hline Mujeres & $64,4 \%$ & $30,69 \%$ & $5,94 \%$ & $1,98 \%$ \\
\hline Hombres & $44,44 \%$ & $36,11 \%$ & $8,33 \%$ & $2,78 \%$ \\
\hline $\begin{array}{l}\text { (4) Sospecha muchas veces } \\
\text { que le soy infiel }\end{array}$ & $79,56 \%$ & $8,76 \%$ & $6,57 \%$ & $2,19 \%$ \\
\hline Mujeres & $82,18 \%$ & $8,91 \%$ & $5,94 \%$ & $1,98 \%$ \\
\hline Hombres & $72,22 \%$ & $8,33 \%$ & $8,33 \%$ & $2,78 \%$ \\
\hline
\end{tabular}

En general, podría decirse que se hallaron frecuencias bajas en perpetración y vivencia de agresiones físicas y sexuales. Sin embargo, las conductas de tipo psicológicas mostraron una mayor prevalencia en las relaciones de jóvenes. En atención a los resultados, destaca que la violencia de tipo psicológico es la más frecuente entre las parejas adolescentes y que coincide con los estudios de Pazos et al. (2014) y de Muñoz-Rivas et al. (20l0), quienes señalaron la violencia verbal como la forma de violencia más utilizada en las relaciones de pareja tanto en chicos como en chicas.Además, en relación con la idea 
Tabla 8. Comportamientos relacionados con el control

\begin{tabular}{|c|c|c|c|c|}
\hline Ítem & I= nunca & $\begin{array}{l}2=\text { pocas } \\
\text { veces }\end{array}$ & $\begin{array}{l}3 \text { = de vez } \\
\text { en cuando }\end{array}$ & $\begin{array}{c}4=\mathrm{a} \\
\text { menudo }\end{array}$ \\
\hline \multicolumn{5}{|c|}{ Comportamientos hacia la pareja } \\
\hline $\begin{array}{l}\text { (I) He impedido que } \\
\text { viese a sus amigos }\end{array}$ & $91,24 \%$ & $5,84 \%$ & ,73\% & $2,19 \%$ \\
\hline Mujeres & $92,08 \%$ & $4,95 \%$ & ,99\% & $1,98 \%$ \\
\hline Hombres & $88,89 \%$ & $8,33 \%$ & $2,78 \%$ & $0 \%$ \\
\hline $\begin{array}{l}\text { (2) He intentado } \\
\text { restringir el } \\
\text { contacto con } \\
\text { su familia }\end{array}$ & $97,81 \%$ & $2,19 \%$ & $0 \%$ & $0 \%$ \\
\hline Mujeres & $99,01 \%$ & ,99\% & $0 \%$ & $0 \%$ \\
\hline Hombres & $94,44 \%$ & $5,56 \%$ & $0 \%$ & $0 \%$ \\
\hline $\begin{array}{l}\text { (3) Insisto en saber } \\
\text { en dónde está en } \\
\text { todo momento }\end{array}$ & $74,45 \%$ & $16,79 \%$ & $6,57 \%$ & $2,19 \%$ \\
\hline Mujeres & $77,23 \%$ & $18,81 \%$ & $2,97 \%$ & ,99\% \\
\hline Hombres & $66,67 \%$ & $11,11 \%$ & $16,67 \%$ & $5,59 \%$ \\
\hline $\begin{array}{l}\text { (4) Miro su teléfono } \\
\text { para ver quien le } \\
\text { llama }\end{array}$ & $77,37 \%$ & $15,33 \%$ & $4,38 \%$ & $1,46 \%$ \\
\hline Mujeres & $80,2 \%$ & $12,87 \%$ & $3,96 \%$ & ,99\% \\
\hline Hombres & $69,44 \%$ & $22,22 \%$ & $5,56 \%$ & $2,78 \%$ \\
\hline $\begin{array}{l}\text { (5) Miro su correo/ } \\
\text { mail }\end{array}$ & $96,34 \%$ & $\mathrm{I}, 46 \%$ & $2,19 \%$ & $0 \%$ \\
\hline Mujeres & $95,05 \%$ & I,98\% & $2,97 \%$ & $0 \%$ \\
\hline Hombres & $100 \%$ & $0 \%$ & $0 \%$ & $0 \%$ \\
\hline
\end{tabular}

Comportamientos de la pareja

\begin{tabular}{|c|c|c|c|c|}
\hline $\begin{array}{l}\text { (I) Ha impedido } \\
\text { que viese a mis } \\
\text { amigos }\end{array}$ & $92,7 \%$ & $3,65 \%$ & ,73\% & $0 \%$ \\
\hline Mujeres & $94,06 \%$ & $3,96 \%$ & ,99\% & $0 \%$ \\
\hline Hombres & $88,89 \%$ & $2,78 \%$ & $0 \%$ & $0 \%$ \\
\hline $\begin{array}{l}\text { (2) Ha intentado } \\
\text { restringir el } \\
\text { contacto con } \\
\text { mi familia }\end{array}$ & $94,16 \%$ & $2,19 \%$ & ,73\% & $0 \%$ \\
\hline Mujeres & $98,02 \%$ & ,99\% & $0 \%$ & $0 \%$ \\
\hline Hombres & $83,33 \%$ & $5,56 \%$ & $2,78 \%$ & $0 \%$ \\
\hline $\begin{array}{l}\text { (3) Insiste en saber en } \\
\text { dónde estoy en } \\
\text { todo momento }\end{array}$ & $77,37 \%$ & $13,87 \%$ & $3,65 \%$ & $2,19 \%$ \\
\hline Mujeres & $84,16 \%$ & II,88\% & $1,98 \%$ & ,99\% \\
\hline Hombres & $58,33 \%$ & $19,44 \%$ & $8,33 \%$ & $5,56 \%$ \\
\hline $\begin{array}{l}\text { (4) Mira mi teléfono } \\
\text { para ver quién } \\
\text { me llama }\end{array}$ & $72,26 \%$ & $17,52 \%$ & $4,38 \%$ & $2,19 \%$ \\
\hline Mujeres & $76,24 \%$ & $16,83 \%$ & $2,97 \%$ & I,98\% \\
\hline Hombres & $61,11 \%$ & $19,44 \%$ & $8,33 \%$ & $2,78 \%$ \\
\hline $\begin{array}{l}\text { (5) Me mira el } \\
\text { correo/mail }\end{array}$ & $94,89 \%$ & $2,19 \%$ & $0 \%$ & $0 \%$ \\
\hline Mujeres & $97,03 \%$ & I,98\% & $0 \%$ & $0 \%$ \\
\hline Hombres & $88,89 \%$ & $2,78 \%$ & $0 \%$ & $0 \%$ \\
\hline
\end{tabular}

según la cual "las mujeres ejercen más violencia de tipo psicológico que los hombres”, se halló en los resultados que las mujeres reportaron haber ejercido más este tipo de violencia que los hombres, lo que coincide también con estudios anteriores (Pazos et al., 2014; Rodríguez, 2015).

Por otra parte, en atención a la violencia de tipo físico y sexual se encontró una prevalencia muy baja en las relaciones de pareja de los jóvenes objeto de estudio, de manera que este tipo de comportamientos son poco frecuentes en la relación. Las investigaciones que analizan este fenómeno suelen encontrar que las mujeres son más vulnerables a este tipo de maltrato, aunque en el caso de conductas de control se hallaron diferencias significativas.

En la exploración de las conductas de celos y control en la relación se halló una prevalencia más alta que en la violencia física y sexual, pero inferior a las conductas de violencia psicológica. Es preciso destacar la diferencia que existe entre los celos y los celos patológicos. Los celos normales son reacciones normales que se asocian al miedo de perder a la pareja, sin embargo, los celos patológicos responden al patrón de ideas irracionales construidas sobre la base de sospechas infundadas. Las personas que sufren de celotipia suelen perder el control y desarrollar, en el peor de los casos, conductas agresivas hacia la pareja.

Los resultados de esta investigación señalan una alta prevalencia de la violencia bidireccional (ejercida y sufrida) de conductas violentas, sobre todo de naturaleza psicológica tanto de hombres hacia mujeres como de mujeres hacia hombres, lo que coincide con otros estudios similares (Fernández-González et al., 2014;Viejo, 2014; Volpe et al., 20।4), quienes indican que el tipo de violencia de este tipo de relaciones es de carácter bidireccional.

En general, en España, la violencia de pareja entre jóvenes es un problema que genera alarma social por la asociación que existe con la violencia de género y, más preocupante, con los homicidios de pareja. Aunque en esta investigación se observan bajas tasas de prevalencia, despierta un interés inquietante como para seguir en la línea de su estudio sistemático para conocer mejor los patrones, los factores de riesgo y los elementos clave que permitan diseñar estrategias de intervención dirigidas a reducir e incluso erradicar el fenómeno.

En España sigue el aumentó el número de jóvenes que sufren algún tipo de abuso por parte de sus parejas. Los 
datos obtenidos en este estudio están en la misma línea de la investigación de Muñoz y Echeburúa (2015), en la que se analizaron creencias y actitudes sobre los roles de género en jóvenes estudiantes con el propósito de comprobar si existían diferencias en función del nivel de estudio y del sexo. Se encontró que las chicas presentaban más actitudes sexistas relacionadas con el contexto laboral y más actitudes trascendentes en el ámbito social, mientras que en los chicos los resultados no fueron significativos (Muñoz y Echeburúa 20I5).

En cuanto a las limitaciones de este estudio, la más importante es el criterio de extracción y el tamaño de la muestra (por accesibilidad), lo que no permite generalizar ni transferir los resultados a otras poblaciones. Por el mismo motivo, el tamaño de la muestra imposibilita el estudio de las variables contextuales, familiares y personales en relación con la violencia. Por otra parte, debido a su tamaño no se realizaron correlaciones. Los resultados hallados deben considerarse una mera aproximación al fenómeno de la violencia en las relaciones de pareja de jóvenes. En estudios futuros es importante el análisis de estas variables con una muestra mayor para aportar resultados más significativos y estar en capacidad de estudiar la etiología de la violencia en las relaciones de pareja de manera más profunda y rigurosa. De igual forma, sería interesante estudiar si existen diferencias significativas en función de la nacionalidad, ya que en un reciente estudio se encontraron diferencias entre maltratadores de nacionalidad española con respecto a los inmigrantes (Fernández-Montalvo, Echauri, Martínez y Azcárate, 20I I).

Este acercamiento a la violencia en las relaciones de pareja de jóvenes pretende dar a conocer y cuantificar el fenómeno de la violencia entre los jóvenes, el cual es un grave problema social que tiene graves consecuencias a corto y largo plazo en la salud de las víctimas. Es de gran relevancia tener la capacidad de identificar variables que rodean a la violencia de pareja en esta etapa evolutiva en la que se crean y desarrollan tanto la personalidad como los patrones relacionales en un contexto íntimo de pareja. Es necesario frenar en estas etapas los estereotipos de género, el uso de la violencia para resolver conflictos, así como los mitos del amor romántico que aún persisten. El propósito final es ayudar a la creación de políticas de prevención que se traduzcan en acciones dirigidas a solucionar el modo de resolver conflictos personales. Esto se convertiría en grandes beneficios para las víctimas directas e indirectas en particular, y para la sociedad en general.

\section{Referencias}

Celis-Sauce, A., \& Rojas-Solís, J. L. (2015). Adolescentes mexicanos como víctimas y perpetradores de violencia en el noviazgo. ReiDoCrea, 4, 60-65.

Cortés, M. L., Bringas, C., Rodríguez-Franco, L., Flores, M., Ramiro-Sánchez,T., \& Rodríguez, F.J. (2014). Unperceived dating violence among Mexican students. International Journal of Clinical and Health Psychology, I4(I), 39-47.

Fernández-González, L., O'Leary K. D., \& Muñoz-Rivas, M. J. (2014). Age-related changes in dating aggression in Spanish high school students. Journal of Interpersonal Violence, 29(6), II32-1I52.

Fernández-Montalvo, J., Echauri, J., Martínez, M., \& Azcárate, J. M. (20II). Violencia de género e inmigración: un estudio exploratorio del perfil diferencial de hombres maltratadores nacionales e inmigrantes. Behavioral Psychology/ Psicología Conductual, 19(2), 439-452.

Fry, D., Davidson, L. L., Rickert, V., \& Lessel, H. (2008). Partners and peers: sexual and dating violence among NYCYouth. New York City Alliance Against Sexual Assault.

González-Ortega, I., Echeburúa, E., \& de Corral, P. (2008).Variables significativas en las relaciones violentas en parejas jóvenes: Una revisión. Behavioral Psychology, I6(2), 2017-225.

Lazarevich, I., Irigoyen, M. E., Sokolova, A. V., \& Delgadillo, H. J. (2012). Violencia en el noviazgo y salud mental en estudiantes universitarios mexicanos. Global Health Promotion, 20(3), 94-103.

López-Cepero, J., Rodríguez, L., Rodríguez, F. J., \& Bringas, C. (20I4).Violencia en el noviazgo: revisión bibliográfica y bibliométrica. Arquivos Brasileiros de Psicología, 66, I- 17.

Martínez, J., Vargas, R., \& Novoa, M. (2016). Relación entre la violencia en el noviazgo y observación de modelos parentales de maltrato. Psychologia: Avances de la Disciplina, IO(I), 101 .

Mohamed, L., Herrera, L., \& Carracedo, S. (20I4).Violencia de pareja en jóvenes estudiantes universitarios de diferente origen cultural. Revista de Edução e Humanidades, 5, 223236.

Muñoz-Rivas, M.J., Gámez-Guadix, M., Graña,J.L., \& Fernández, L. (2010). Violencia en el noviazgo y consumo de alcohol y drogas ilegales entre adolescentes y jóvenes españoles. Adicciones, 22, 125-1 34.

Muñoz-Rivas, M. J., Graña, J. L., O’Leary, K. D., \& González, M. P. (2007a). Aggression in adolescent dating relationships: prevalence, justification, and health consequences. Journal of Adolescent Health, 40, 298-304. 
Muñoz, J. M., \& Echeburúa, E. (20I6). Diferentes modalidades de violencia en la relación de pareja implicaciones para la evaluación psicológica forense en el contexto legal español. Anuario de Psicología. 26, 2-12

Palmetto, N., Davidson, L. L., Breitbart,V., \& Rickert,V. I. (20I3). Predictors of physical intimate partner violence in the lives of young women: victimization, perpetration and bidirectional violence. Violence and Victims, 28(I), I03-12I.

Pazos, M., Oliva, A., \& Gómez, A. (2014).Violencia en relaciones de pareja de jóvenes y adolescentes. Revista Latinoamericana de Psicología, 46(3), I48-I59.

Ramírez, C., \& Núñez, L. (2010).Violencia en la relación de noviazgo en jóvenes universitarios: un estudio exploratorio. Enseñanza e Investigación en Psicología, 15(2), 273-283.

Rodríguez, R., Riosvelasco, L., \& Castillo, N. (2018).Violencia en el noviazgo, género y apoyo social en jóvenes universitarios. Escritos Psicológicos, I I (I), I-9.

Rodríguez, S. (2015). Violencia en las parejas jóvenes: estudio preliminar sobre su prevalencia y motivos. Pedagogía Social. Revista Interuniversitaria, 25, 25 I-275.
Rojas-Solís, J. L. (2013). Violencia en el noviazgo de universitarios en México: una revisión. Revista Internacional de Psicología, I2(2), I-3I.

Sebastián, J., Ortiz, B., Gil, M., Gutiérrez, M., Hernáiz,A., \& Hernández, J. (2010). La violencia en las relaciones de pareja de los jóvenes. ¿Hacia dónde caminamos? Clínica Contemporánea, I (2), 7 I-83.

Valdivia, M. P., \& González, L.A. (20 |4).Violencia en el noviazgo y pololeo: una actuación proyectada hacia la adolescencia. Revista de Psicología, 32(2), 329-355.

Viejo, C. (20|4).Violencia física en las relaciones sentimentales adolescentes: hacia la comprensión del fenómeno. Infancia y Aprendizaje:Journal for the Study of Education and Development, 37(4), 785-8I5.

Volpe, E. M., Morales-Alemán, M. M., \& Teitelman, A. M. (2014). Urban adolescent girls' perspectives on romantic relationships: initiation, involvement, negotiation, and conflict. Issues in Mental Health Nursing, 35, 776-790. 